\title{
Replacement Algorithm of Virtual Service Resources with Dynamic Resource Allocation Based on Traffic Change in Virtual Networks
}

\author{
Sakie Horiuchi*, Takuji Tachibana \\ Graduate School of Engineering, University of Fukui, Fukui, Japan \\ *Corresponding author: sakie-h@network.fuis.u-fukui.ac.jp
}

Received August 02, 2018; Revised September 09, 2018; Accepted September 26, 2018

\begin{abstract}
Multiple virtual networks are constructed by using network virtualization technology on a substrate network with network resources. By using virtual service resources, a particular service can be provided with users over each virtual network. Moreover, network virtualization for mobile users has been expected in the future network service. In mobile virtual networks, mobile user moves from a node to other nodes and connects to other access points frequently. Therefore, the traffic on each link and each node in the virtual network changes. Because the quality of the service depends on the amount of traffic on links and nodes, the quality of services changes according to the user's movement. This paper proposes replacement algorithm of virtual service resources with dynamic resource allocation based on traffic change for mobile applications in virtual networks. In our proposed method, the amount of resources for each node and that for each link are changed based on the traffic change. Moreover, the virtual service resource moves to the adjacent node from a node, and this replacement is repeated until the quality of service satisfies the requested quality. With our proposed method, it is expected that the quality of service can be maintained for mobile users even if the amount of traffic changes. We evaluate the performance of our proposed method with simulation. In numerical examples, it is shown that our proposed method is effective when a larger number users move and the amount of traffic changes.
\end{abstract}

Keywords: virtual network, virtual service resource, traffic change, mobile user

Cite This Article: Sakie Horiuchi, and Takuji Tachibana, "Replacement Algorithm of Virtual Service Resources with Dynamic Resource Allocation Based on Traffic Change in Virtual Networks." Journal of Computer Networks, vol. 5, no. 1 (2018): 8-15. doi: 10.12691/jcn-5-1-2.

\section{Introduction}

Currently, network virtualization technology has been utilized actively all over the world $[1,2,3]$. Much focus is being placed on network virtualization technology as one of new generation network technologies $[4,5,6,7]$. Multiple virtual networks are constructed by using the network virtualization technology on a substrate network with network resources such as memory, CPU, and bandwidth $[8,9,10]$. Virtual networks have already been used in several use cases [11,12], and it is expected that network virtualization technology can be utilized in more situations. As a system that utilizes the network virtualization, a system where a service provider constructs virtual networks over a substrate network and provides them with users has been utilized. In such a system, a constructed virtual network is independent with other constructed virtual networks. Therefore, the virtual network is not affected by other virtual networks. As a result, each user of virtual network can provide a different service over each virtual network [13].

In the system with network virtualization, each virtual network has to be constructed by utilizing network resources of a substrate network effectively. This is because the virtual networks are constructed by sharing resources of the substrate network that are finite. Moreover, in a recent communication network, many failures such as packet loss and congestion in network occur due to the growth of data traffic in the communication network [14]. Hence, the virtual networks are expected to be constructed so as to increase the robustness of virtual networks. For this system, some methods have been proposed. In the virtual network construction, data packets can be transmitted over each virtual network even if failures occur. By utilizing these methods, each virtual network can keep the data transmission service even if a failure of single link of the substrate network occurs. Moreover, virtual network construction methods for robust substrate networks have been proposed in [15], [16] and [17]. In these methods, virtual networks are constructed over a substrate network while utilizing conventional data transmission services such as existing Internet services in the substrate network. Especially, the conventional services are high priority classes and qualities of those services should not be degraded. In such a case, virtual networks are expected to be constructed over the substrate network so as not to 
degrade the qualities. Therefore, these proposed methods utilize an admission control for the virtual network construction, and virtual networks can be constructed based on a construction condition.

On the other hand, over each virtual network, a particular service can be operated and provided with users. A server for the particular service is called virtual service resource, and users can access the virtual service resources to utilize the network service. Moreover, network virtualization for mobile users have been studied in the future network service [18]. In such virtual networks where mobile users utilize virtual service resources, mobile user moves from a node to other nodes and connects to other access points frequently. Therefore, the traffic on each link and each node in the virtual network changes. Because the quality of the service depends on the amount of traffic on links and nodes, the quality of services changes according to the user's movement. If the amount of traffic becomes small (large) on a link, the quality of the service that passes through the link becomes high (low).

When some users who have utilized a virtual service resource move to other place, a new node may be added into the virtual network. Moreover, the amount of traffic on a node changes, and hence the amount of traffic on a link may change. In order to maintain the quality of services, the amount of resources on links and nodes should be changed based on the traffic change. In addition, the place of virtual service resources should also be changed based on the traffic change.

In this paper, we propose replacement algorithm of virtual service resources with dynamic resource allocation based on traffic change for mobile applications in virtual networks. Our proposed method consists of 1) dynamic resource allocation based on traffic change and 2) dynamic replacement of virtual service resources based on traffic change. In the dynamic resource allocation, the amount of resources for each node and that for each link are changed based on the traffic change. By this resource allocation, the more (less) amount of node and link resources can be allocated when the amount of traffic increases (decreases). On the other hand, in this dynamic replacement of virtual service resources, the virtual service resource moves to the adjacent node from a node. This replacement is repeated until the quality of service satisfies the requested quality. With our proposed method, it is expected that the quality of service can be maintained for mobile users even if the amount of traffic changes. We evaluate the performance of our proposed method with simulation and investigate the effectiveness of the proposed method.

The rest of this paper is organized as follows. Section 2 introduces how to construct virtual network. Section 3 explains the detail of our proposed method. Section 4 shows some numerical examples and Section 5 denotes conclusions.

\section{Existing Virtual Network Construction Considering Network Robustness}

In this section, we describe how to construct virtual networks based on the robustness of physical network.

\subsection{Virtual Network Construction Procedure}

At first, we explain a procedure of virtual network construction $[16,17]$. In the following, we assume that each virtual network is provided with the service provider by the telecommunications carrier that manages a substrate network. When a telecommunications carrier receives a request of virtual network construction from a service provider, a virtual network is constructed according to the following procedure. Figure 1 also shows the procedure of virtual network construction.

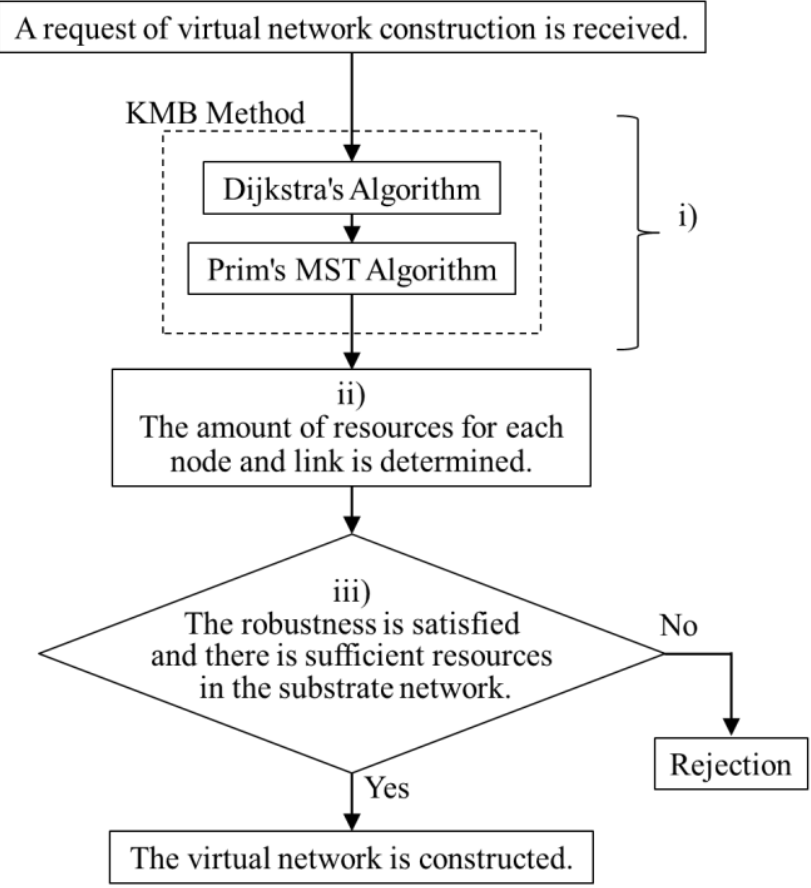

Figure 1. Procedure of virtual network construction

i) At first, telecommunications carrier designs the topology of virtual network with KMB algorithm [19].

ii) Then, the telecommunications carrier determines the amount of resources for each node and each link according to the user's request.

iii) Finally, the virtual network is constructed if the robustness of the virtual network is satisfied and the amount of resources for each node and each link in the substrate network is larger than the amount of resources that are used in the virtual network. Otherwise, the request of virtual network construction is rejected.

The KMB algorithm is a method that combines Dijkstra's algorithm for finding the shortest path and Prim's MST algorithm for finding the minimum spanning tree. At i), the telecommunications carrier receives the virtual network construction request that includes the information about nodes that are used in the virtual network, the shortest path is derived using the Dijkstra's algorithm between arbitrary two requested nodes. Then, a complete graph for the requested nodes is generated. Next, by using Prim's MST algorithm, the topology of the virtual network is designed by finding the minimum tree passing through all the request nodes. Therefore, in this procedure, the topology of virtual network can be designed so that network cost is minimized. 


\subsection{Resource Allocation and Construction Conditions}

In this subsection, we explain the resource allocation at ii) and the construction condition at iii) in the previous subsection.

Now, we consider a virtual network with $N$ nodes. In this network, we denote the $i$ th node as $n_{i}(i=1, \cdots, N)$. Moreover, we denote the number of users that are directly connected to node $n_{i}$ as $u_{i}(i=1, \cdots, N)$. Here, we assume that $u_{i}$ users utilize the virtual service resource $S$. We also represent a link between node $n_{i}$ and $n_{j}$ as $l_{i j}$.

In the following, the amount of traffic from $u_{i}$ users to $S$ is assumed to be $\gamma_{i}=\alpha u_{i}$ where $\alpha$ is a traffic coefficient. In this case, the amount $\gamma_{p q}$ of traffic on link $l_{p q}$ is calculated by

$$
\gamma_{p q}=\sum_{i=1}^{N} \sum_{p q} \gamma_{i}=\sum_{i}^{N} \sum_{i=1} \sum_{p q} \alpha u_{i}
$$

In this equation, $R_{i}$ is a route, that includes some links, from node $n_{i}$ to virtual service resource $S$.

Let the amount $m_{p q}$ of resources on any link $l_{p q}$ in the virtual network satisfy the following inequality (2), the quality of the service can satisfy the requested quality in this virtual network.

$$
\frac{\gamma_{p q}}{m_{p q}} \leq c
$$

As $c$ becomes large, the amount of traffic that can be accommodated in link $l_{p q}$ becomes large.

Here, at iii) in subsection 2.1, the virtual network can be constructed according to the quality of services and the amount of resources. If all the following construction conditions are satisfied, a virtual network can be constructed.

1. Network robustness of substrate network satisfies a required network robustness that is denoted as a threshold for a performance metric [20].

2. The amount of resources for each node and each link in the virtual network is smaller than the amount of resources that can be used in the substrate network.

3. The amount of resource for each link in the virtual network satisfies (2).

If at least one condition cannot be satisfied among the above three conditions, the virtual network is not constructed and the request is rejected.

\section{Replacement Algorithm of Virtual Service Resources with Dynamic Resource Allocation Based on Traffic Change}

In this section, we propose a replacement algorithm of virtual service resources with dynamic resource allocation for virtual networks where mobile users utilize network services. By using this method, it is expected that users can utilize the virtual service resources appropriately.

\subsection{Overview}

In this subsection, we denote an overview of our proposed method. Here, our proposed method can be utilized for virtual networks where virtual service resources have been utilized over a substrate network (see Figure 2). In this paper, we call a server for the particular service virtual service resource.

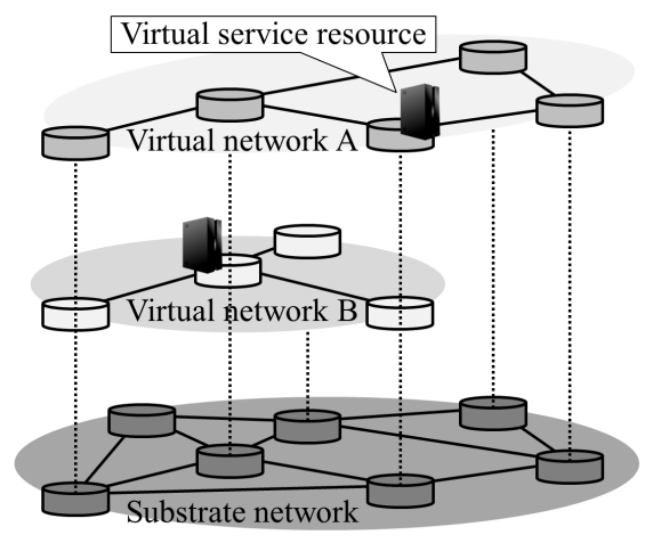

Figure 2. Virtual service resource in virtual networks

Now, we consider a case where the number of users changes or a new node is added due to the user's movement. Depending on such a user's movement, the amount of traffic in virtual network changes drastically. Therefore, in our proposed method, the virtual network services can be replaced and the amount of network resources in virtual networks can be changed according to the traffic change.

The procedure of our proposed method is shown in the following. Moreover, Figure 3 also shows a flowchart of our proposed method.

i) If there is no new node in the virtual network due to the user's movement, go to Step ii). Otherwise, go to Step iv).

ii) The amount of resources for each node and each link is changed (see subsection 3.2). Then, go to Step iii).

iii) If this virtual network satisfies all the three construction conditions shown in subsection 2.2, the virtual network is constructed. Otherwise, the virtual network cannot be utilized because the quality of services cannot be satisfied. Of course, this virtual network can be used if users can accept the low service quality but this is out of scope in this paper.

iv) A new topology of the virtual network is redesigned because the new node is added (see subsection 3.3). Then go to Step v).

v) If the virtual network satisfies construction conditions in subsection 2.2 , the virtual network is constructed. Otherwise, go to Step vi).

vi) The virtual service resource is replaced in the virtual network (see subsection 3.4). Then, go to Step vii).

vii) If the virtual network satisfies all the three construction conditions in subsection 2.2, the virtual network is constructed. Otherwise, the virtual network cannot be utilized because the quality of services cannot be satisfied. 


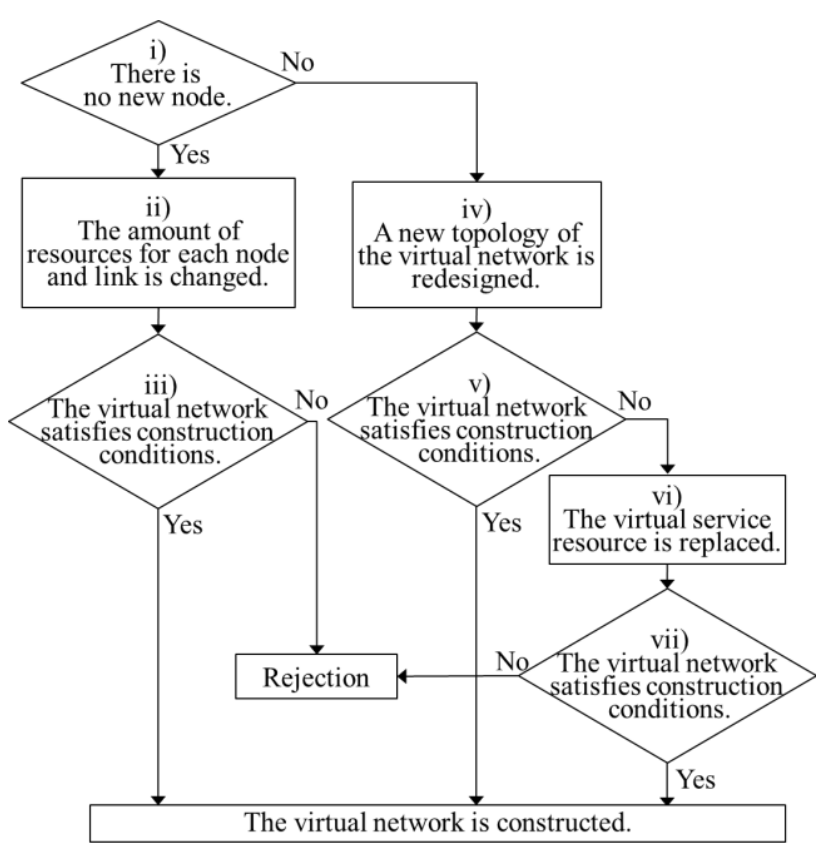

Figure 3. Flowchart of proposed method

In this procedure, at Step ii), the amount of resources for each node and each link is changed with dynamic resource allocation. This is explained in subsection 3.2. At Step iv), a new topology is designed with shortest path betweenness centrality. This is explained in subsection 3.3. At Step vi), the virtual service resource is replaced with our proposed method. The detail of this replacement is explained in subsection 3.4 .

\subsection{Dynamic Resource Allocation Based on Traffic Change}

In this subsection, we explain our proposed dynamic resource allocation that is used at Step ii).

In the following, we consider a virtual network with $N$ nodes as is the case with the network shown in subsection 2.2. In this network, we denote the $i$ th node as $n_{i}(\mathrm{i}=1, \cdots N)$ and the amount of available resources for node $n_{i}$ is represented as $m_{i}$. Moreover, $l_{i j}$ denotes a link between node $n_{i}$ and $n_{j}$ and the amount of resources for link $l_{i j}$ is represented as $m_{i j}$. The number of users that are directly connected to node $n_{i}$ is denoted as $u_{i}(i=$ $1, \cdots, N)$, and $u_{i}$ users utilize the virtual service resource $S$. In this network, the amount of traffic from $u_{i}$ users to $S$ is denoted as $\gamma_{i}=\alpha u_{i}$, and the amount of traffic on link $l_{p q}$ is represented as $\gamma_{p q}$.

Our proposed dynamic resource allocation can increase or decrease the amount of resources for each link and each node based on the traffic change. Here, when the amount of traffic for node $n_{i}$ changes from $\gamma_{i}$ to $\gamma_{i}{ }^{\prime}$, by using the proposed dynamic resource allocation, the amount $m_{i}$ of resources for node $n_{i}$ is changed into $m_{i}^{\prime}$ as follows.

$$
m_{i}^{\prime}=\frac{m_{i} \gamma_{i}^{\prime}}{\gamma_{i}} .
$$

If $\gamma_{i}$ is larger (smaller) than $\gamma_{i}{ }^{\prime}$, the amount of resources for $n_{i}$ increases (decreases) from $m_{i}$ to $m_{i}^{\prime}$ as shown in (3).
On the other hand, for link $l_{i j}$, we assume that the amount of traffic changes from $\gamma_{i j}$ to $\gamma_{i j}{ }^{\prime}$. In this case, by using this method, the amount $m_{i j}$ of resources for link $l_{i j}$ is changed into $m_{i j}^{\prime}$ as follows.

$$
m_{i j}^{\prime}=\frac{m_{i j} \gamma_{i j}^{\prime}}{\gamma_{i j}} .
$$

As is the case with the node resources, the amount of link resources increases (decreases) if the amount of traffic becomes large (small).

\subsection{Topology Redesign with Shortest Path Betweenness Centrality}

In this subsection, we explain a topology redesign of virtual network with shortest path betweenness centrality that is used at Step iv).

Now, we consider a virtual network where a new node has to be added. Here, the new added node is denoted as $n_{k}$. In the proposed method, in order to add $n_{k}$ into the virtual network, a new topology is redesigned with shortest path betweenness centrality [21].

In this redesign, at first, $n_{k}$ is defined as the start node. Moreover, let $N_{\text {start }}$ be a set of start node and $n_{k}$ is added into $N_{\text {start }}$. Shortest path betweenness centrality of each link is also calculated (see Figure 4 ). Then, $n_{k}$ is added into the virtual network according to the following steps.

i) For the adjacent nodes of the start node in the substrate network, it is checked whether some adjacent nodes have been included in the virtual network. If adjacent node $n_{e}$ has been included in the virtual network, a link between $n_{e}$ and the start node is added into the virtual network. Moreover, all nodes in $N_{\text {start }}$ are added into the virtual network. Because a new topology was redesigned, this procedure finishes. Otherwise, go to Step ii).

ii) Among links that are connected to the start node, a link whose shortest path betweenness centrality is the largest is selected. When the selected link is connected to $n_{a}$ in addition to the start node, node $n_{a}$ is set to the start node and $n_{a}$ is added into $N_{\text {start }}$. Then, return to Step i).

Figure 4 shows an example of our proposed topology redesign. In this figure, nodes $n_{2}, n_{3}, n_{4}$ and $n_{5}$ have already been included in the virtual network, and solid lines denote links in the virtual network. For this virtual network, node $n_{8}$ would like to be added into the virtual network as a new node. In addition, dot lines denote links in the substrate network and the shortest path betweenness centrality is shown on each link. Now, $n_{8}$ is the start node. At first, no adjacent node of $n_{8}$ is included in the virtual network. Therefore, the adjacent link $l_{68}$ is selected depending on the shortest path betweenness centrality. Then, $n_{6}$ is set to the start node. Here, the adjacent node $n_{2}$ of $n_{6}$ has been included in the virtual network. Therefore, a new topology is redesigned by adding $n_{6}, n_{8}$, $l_{68}$, and $l_{26}$ into the virtual network.

After the new topology is redesigned, the amount of resources for each link and each node is decided according to (3) and (4) in subsection 3.2. 


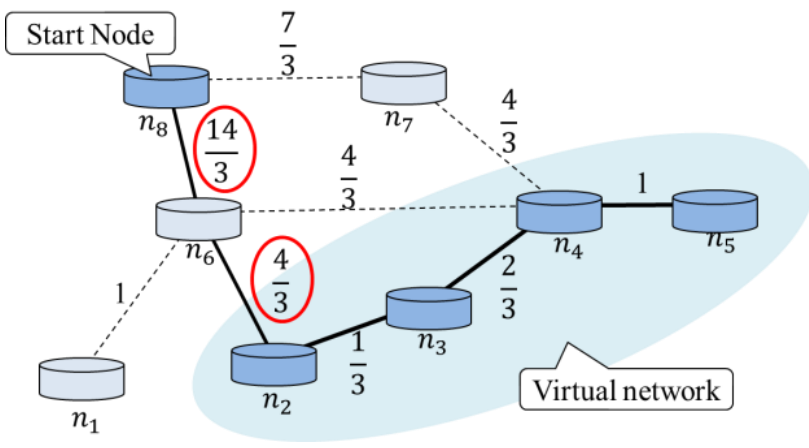

Figure 4. An example of our proposed topology redesign

\subsection{Dynamic Replacement of Virtual Service Resources for a Virtual Network}

Finally, in this subsection, we explain dynamic replacement of virtual service resource that is used in Step vi).

In previous subsection, new node can be added into the virtual network by our proposed method. However, with the dynamic resource allocation, construction conditions that are shown in subsection 2.2 may not be satisfied. Here, we denote a node where a virtual service resource has been placed as $n_{c}$. Therefore, in this case, virtual service resource is replaced by using the proposed dynamic replacement according to the following procedure (see Figure 5).

i) Both the shortest path and the number of hops from the new added node $n_{k}$ to $n_{c}$ are derived. Then, go to Step ii).

ii) Along the shortest path between $n_{k}$ and $n_{c}$, the virtual service resource moves to the adjacent node $n_{a}$ towards $n_{k}$ by one hop. Then, $n_{a}$ is set to $n_{c}$ and go to Step iii).

iii) If the quality of provided service can be satisfied, this algorithm finishes. If the quality is not satisfied and the number of hops between $n_{k}$ and $n_{c}$ is larger than one, return to Step ii). Otherwise, the dynamic replacement of virtual network services fails.

By using this dynamic replacement, the amount of traffic on the route from the new added node to the virtual service resource can be reduced.

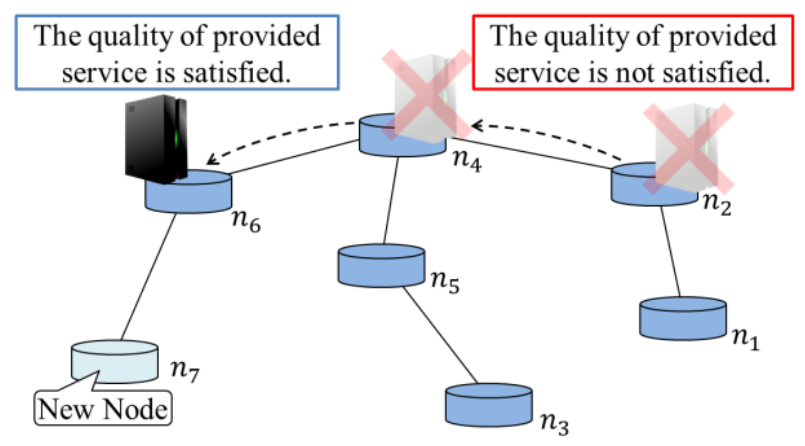

Figure 5. Example of our proposed dynamic replacement of virtual service resource

\section{Numerical Example}

In this section, we evaluate the performance of our proposed method with simulation.

\subsection{Simulation Model}

In this subsection, we explain our simulation model where the performance of our proposed method is evaluated.

Figure 6 shows a substrate network.

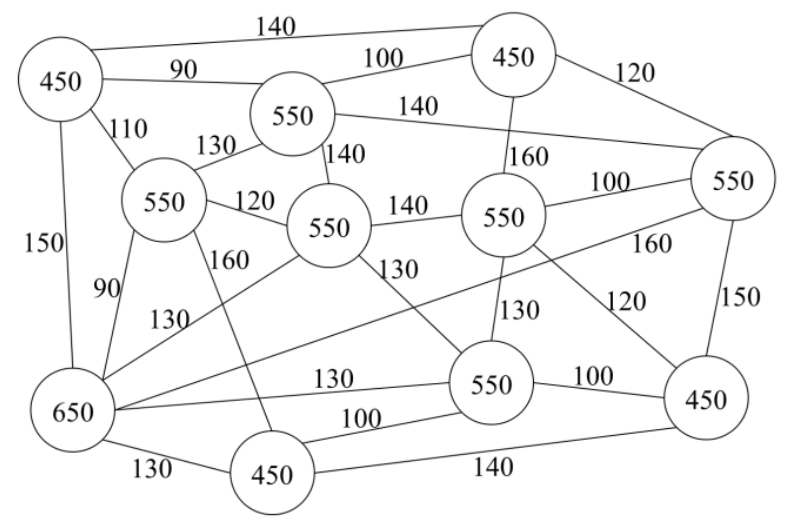

Figure 6. Network Topology

In this substrate network, the amount of resources for each node is from 450 to 650 and the amount of resources for each link is from 90 to 160 . For this substrate network, we assume that a new request for a virtual network construction arrives according to a Poisson process with rate $\lambda_{1}$. This request includes the information on $H$ nodes $(H \leq 3)$ and the amount of resources that are needed for the constructed virtual network. The number $u_{i}$ of users for node $n_{i}$ is determined at random among $[1,20]$. Upon receiving a new request of virtual network, a virtual network is constructed with KMB algorithm [19]. Then, a virtual service resource is placed at random, and the amount of resources for nodes and links are allocated based on the amount of traffic. When a new virtual network is constructed, it is checked whether the virtual network satisfies the construction conditions shown in subsection 2.2. We assume that the utilization time of the virtual network follows an exponential distribution with rate 1.0 .

After a virtual network is constructed, the amount of traffic in the virtual network changes according to the exponential distribution with rate $\lambda_{2}$. Here, a new node is added into the virtual network with probability $\beta$ and the amount of traffic changes with probability $1-\beta$. When the amount of traffic changes, two nodes are randomly selected in the virtual network and 1 to 5 users move between the two nodes. On the other hand, when a new node is added into the virtual network, the number of users for the added node is determined at random among $[1,20]$.

In the following, we evaluate the effectiveness of our proposed method with simulation. We also evaluate the performance of a method where a new topology is redesigned with random walk algorithm instead of shortest path betweenness centrality described in subsection 3.3. This method is denoted as Random walk method. We also evaluate a method where the virtual service resource can be moved to an adjacent node only once. This method is denoted as Move once method. Note that in our proposed method, the virtual service resource can be moved toward the new added node shown in subsection 3.3. In addition, we evaluate a method where the virtual service resource 
can be replaced at random. This method is denoted as Random method.

\subsection{Impact of the Traffic Changing Rate}

In this subsection, we investigate the impact of traffic changing rate $\lambda_{2}$ on the performance of our proposed method. In the following, the request arrival rate $\lambda_{1}$ is equal to 1.0 and probability $\beta$ is equal to 0.5 .

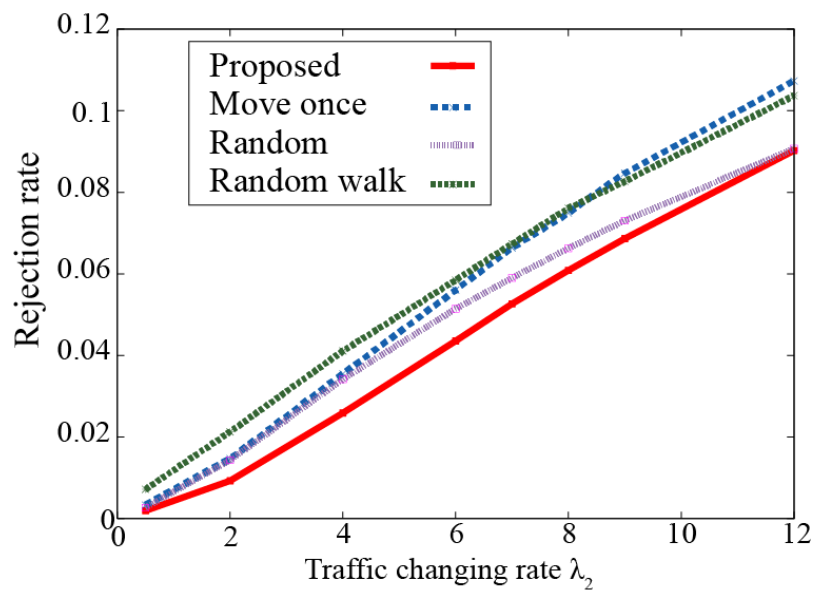

Figure 7. Rejection rate of virtual networks vs. traffic changing rate $\lambda_{2}$

Figure 7 shows the rejection rate of virtual networks against the traffic changing rate $\lambda_{2}$. In this figure, we can find that the rejection rate for the four methods increases as the traffic changing rate $\lambda_{2}$ increases. This is because it becomes hard to satisfy the construction conditions due to the user's movement. However, the rejection rate for our proposed method is smaller than those for other methods regardless of the traffic changing rate $\lambda_{2}$. Therefore, our proposed method can construct a larger number of virtual networks and a larger number of users can utilize the virtual network regardless of the traffic change. Therefore, our proposed method is effective when the amount of traffic changes.

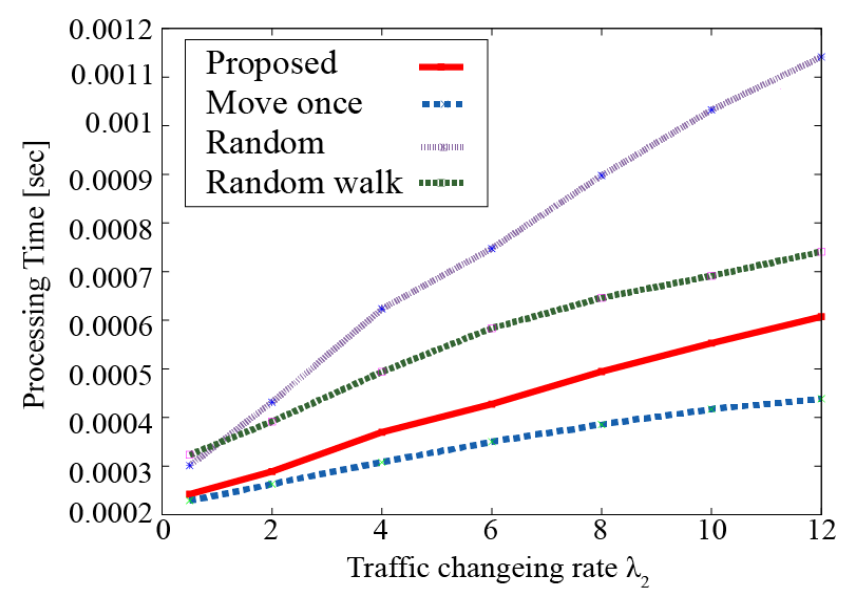

Figure 8. Processing time vs traffic changing rate $\lambda_{2}$

Figure 8 shows the processing time for the virtual network reconstruction that includes the topology redesign and the replacement of virtual service resource against the traffic changing rate $\lambda_{2}$. From this figure, we can find that the processing time of our proposed method is smaller than those for move once method and random move method regardless of the traffic changing rate $\lambda_{2}$. Therefore, our proposed method can reconstruct the virtual network quickly even when the amount of traffic changes.

From these results, by using our proposed method, a larger number of virtual networks can be utilized when the amount of traffic changes.

\subsection{Impact of Probability $\beta$}

In this subsection, we investigate the impact of probability $\beta$ on the performance of our proposed method. In the following, the request arrival rate $\lambda_{1}$ is equal to 1.0 and the traffic changing rate $\lambda_{2}$ is equal to 1.0.

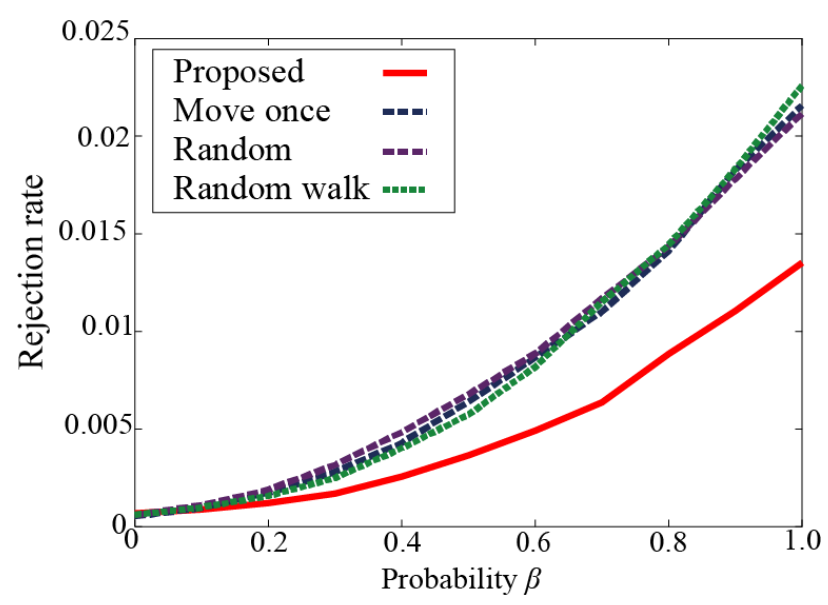

Figure 9. Rejection rate of virtual networks vs. probability $\beta$

Figure 9 shows the rejection rate of virtual networks against probability $\beta$. Here, when $\beta$ is large, a larger amount of new nodes are added in the virtual network. In this figure, we can find that the rejection rate for the four methods increases as $\beta$ becomes large. However, we can find that the rejection rate for our proposed method is smaller than those for other methods regardless of $\beta$. Therefore, our proposed method can construct a larger number of virtual networks even when a larger number of nodes are added in the virtual network due to the user's movement.

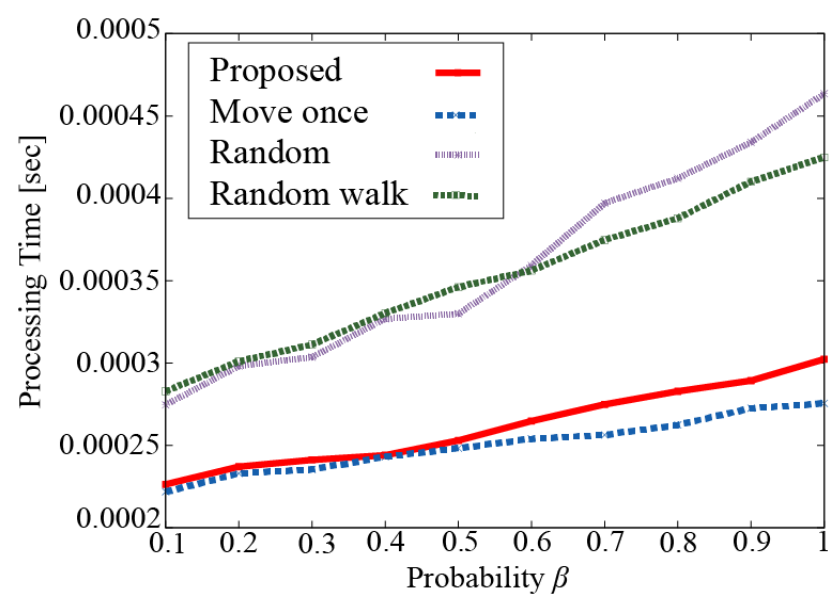

Figure 10. Processing time vs. probability $\beta$

Figure 10 shows the processing time for the virtual network reconstruction that includes the topology redesign and the replacement of virtual service resource against 
probability $\beta$. From this figure, we can find that the processing time for our proposed method is smaller than those for other methods regardless of $\beta$. Therefore, our proposed method can reconstruct the virtual network quickly.

From these results, our proposed method can construct a larger number of virtual networks when a new node is added. Therefore, our proposed method is effective when a larger number users move frequently.

\subsection{Impact of the Request Arrival Rate}

Finally, in this subsection, we investigate the impact of the request arrival rate $\lambda_{1}$ on the performance of our proposed method. In the following, the traffic changing rate $\lambda_{2}$ is equal to 1.0 and probability $\beta$ is equal to 0.5 .

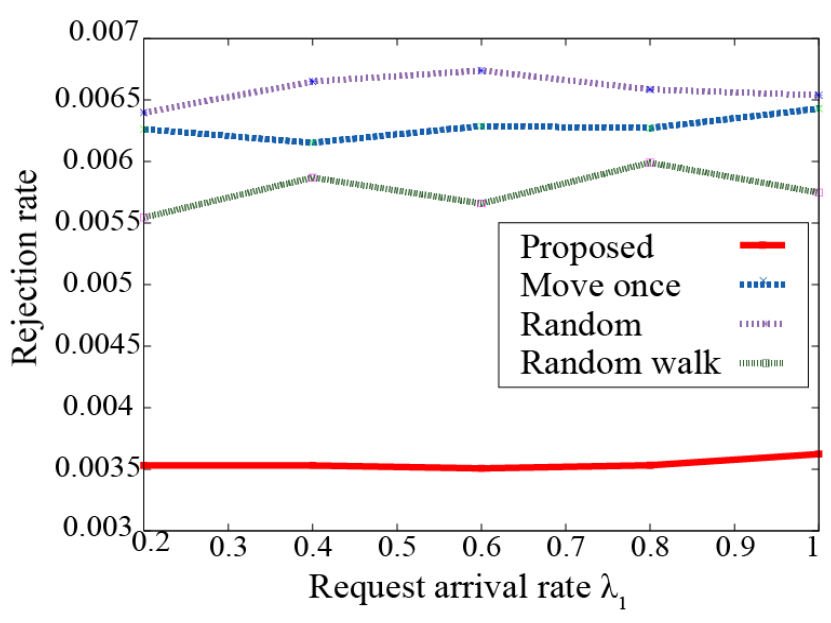

Figure 11. Rejection rate of virtual networks vs. request arrival rate $\lambda_{1}$

Figure 11 shows the rejection rate of virtual networks against the request arrival rate $\lambda_{1}$. In this figure, the rejection rate for each method does not change so much. This means that virtual networks are rejected due to the traffic change even if the arrival rate is small. From this figure, we can find that the rejection rate for our proposed method is smaller than those for other methods regardless of the request arrival rate $\lambda_{1}$. Therefore, our proposed method can construct a larger number of virtual networks regardless of the request arrival rate $\lambda_{1}$.

\section{Conclusion}

In this paper, in order to maintain the quality of provided service, we proposed replacement algorithm of virtual service resources with dynamic resource allocation based on traffic change for mobile applications in virtual networks. In our proposed, the amount of resources for each node and that for each link were changed based on the traffic change. Moreover, the virtual service resource moves to the adjacent node from a node, and this replacement was repeated until the quality of service satisfies the requested quality. We evaluated the performance of our proposed method with simulation. From the performance comparison, we found that our proposed method is effective when a larger number users move and the amount of traffic changes.

\section{Acknowledgements}

We would like to thank Mr. Yuki Koyanagi for giving us his valuable comments and suggestions.

\section{References}

[1] “GENI,” [Online]. Available: http://www.geni.net/. [Accessed: 24 Jul. 2018].

[2] "PlanetLab," [Online]. Available: http://www.planet-lab.org/. [Accessed: 24 Jul. 2018].

[3] "Emulab - Network Emulation Testbed," [Online]. Available: http://www.emulab.net/ [Accessed: 24 Jul. 2018].

[4] Nakao, A., "Network Virtualization as Foundation for Enabling New Network Architectures and Applications," IEICE Transactions on Communications, 93(3). 454-457. Mar. 2010.

[5] Haider, A., Harris, R. J., and Sirisena, H., "Virtualization and New Generation Network Design," in IEEE Australasian Telecommunication Networks and Applications Conference, Christchurch, New Zealand, Nov. 2013, 189-194.

[6] Davalos, E., Aceval, C., Franco, V., and Baran, B., "A MultiObjective Approach for Virtual Network Embedding," in IEEE Latin American Computing Conference, Arequipa, Peru, Oct. 2015, $1-8$.

[7] Drutskoy, D., Keller, E., and Rexford, J., "Scalable Network Virtualization in Software-Defined Networks," IEEE Internet Computing, 17(2). 20-27. Apr. 2013.

[8] Zhu, Y. and Ammar, M. H., "Algorithms for Assigning Substrate Network Resource to Virtual Network Components," in IEEE International Conference on Computer, Barcelona, Spain. Apr. 2006. 1-12.

[9] Yu, M., Yi, Y., Rexford, J., and Chiang, M., "Rethinking Virtual Network Embedding: Substrate Support for Path Splitting and Migration," ACM SIGCOMM Computer Communication Review, 38(2). 17-29. Apr. 2008.

[10] He, J., Zhang-Shen, R., Li, Y., Lee, C. Y., Rexford, J., and Chiang, M., "DaVinci: Dynamically Adaptive Virtual Networks for a Customized Internet," in ACM Conference on Emerging Networking Experiment and Technology, Madrid, Spain. Dec. 2008. 15.

[11] Casado, M. and McKeown, N., "The Virtual Network System," in the 36th SIGCSE Technical Symposium on Computer Science Education, St. Louis, Missouri, USA. Feb. 2005. 76-80.

[12] Iguchi, N., "Virtual IP Network Practice System with Software Agent," in International Conference on Complex, Intelligent, and Software Intensive System, Jul. 2017. 711-720.

[13] Khan, A., Zugenmaier, A., Jurca, D., and Kellerer, W., "Network Virtualization: a Hypervisor for the Internet?," IEEE Communications Magazine, 50(1). 136-143. Jan. 2012.

[14] "Cisco Visual Networking Index," [Online]. Available: https://www.cisco.com/c/ja_jp/solutions/service-provider/visualnetworking-index-vni/index.html. [Accessed: 24 July 2018].

[15] Mori, M., Tachibana, T., Hirata, K., and Sugimoto, K., "A Proposed Topology Design and Admission Control Approach for Improved Network Robustness in Network Virtualization," in IEEE Global Telecommunications Conference, Texas, USA. Dec. 2011.

[16] Urayama, Y. and Tachibana, T., "Virtual Network Construction with K-shortest Path Algorithm and Optimization Problems for Robust Physical Networks," International Journal of Communication Systems, 30(1). 2015.

[17] Urayama, Y., Tsubota, H., and Tachibana, T., "Virtual Network Construction Scheduling Based on Network Criticality and Network Resources for Robust Physical Network," Journal of Internet Technology, 17(2). 359-367. Apr. 2016.

[18] Nakao, A., Du, P., Kiriha, Y., Granelli, F., Gebremariam, A. A., Taleb, T., and Bagaa, M., "End-to-End Network Slicing for 5G Mobile Networks," Journal of Information Processing, 25(1). 153-163. 2017.

[19] Kou, L., Markowsky, G., and Berman, L., "A Fast Algorithm for Steiner Trees," Acta Informatica, 15(2). 141-145. Jun. 1981. 
[20] Tizghadam, A. and Leon-Garcia, A, "Autonomic Traffic Engineering for Network Robustness," IEEE Journal on Selected Areas in Communications, 28(1). 39-50. 2010.
[21] Newman, M. E. J. and Girvan, M., "Finding and Evaluating Community Structure in Networks," Physical Review, E 69. Feb. 2004. 